\title{
Asma e ascaridíase em crianças de 2 a 10 anos de um bairro de periferia
}

\author{
Asthma and ascariasis in children aged two to ten living in a low income suburb
}

\author{
Maria Teresa Nascimento Silva ${ }^{1}$, Jacy Andrade ${ }^{2}$, José Tavares-Neto ${ }^{3}$
}

\section{Resumo}

Objetivo: avaliar a associação entre asma e ascaridíase em crianças do bairro Pedregal, da cidade de Campina Grande (Paraíba), onde são baixos os indicadores de desenvolvimento humano.

Material e método: foi aplicado o questionário padrão do International Study of Asthma and Allergies in Childhood (ISAAC), em 1.095 crianças, mas apenas 742 o responderam e entregaram a amostra fecal para realização do exame parasitológico. A faixa etária das 742 crianças incluídas no estudo transversal foi de 2 a 10 anos de idade. A associação entre asma e ascaridíase foi estudada pelo índice Kappa. As análises das variáveis demográficas e clínicas, comparando os dois grupos, foram realizadas pelo teste do quiquadrado $\left(\chi^{2}\right)$ ou o teste $t$ de Student para os dados quantitativos (contínuos).

Resultados: nas 742 crianças, as proporções dos sexos foram semelhantes: $51,9 \%(n=385)$ do masculino e $48,1 \%(n=357)$ do feminino. Nessas crianças, a prevalência de asma foi de $59,7 \%$ $(\mathrm{n}=443)$ e da ascaridíase de 56,3\% $(\mathrm{n}=418)$, sendo semelhante $\left(\chi^{2}=0,27 ; \mathrm{p}>0,60\right)$ a freqüência de asma no grupo portador de ascaridíase $(60,5 \%)$ ou sem essa helmintíase $(58,6 \%)$.

Conclusão: apesar da elevada freqüência dos agravos estudados, não há aparente associação entre asma e ascaridíase na população estudada.

J Pediatr (Rio J) 2003;79(3):227-32: asma, ascaridíase, criança, prevalência.

1. Professora-adjunta do Dep. de Medicina Interna Social e Preventiva da Faculdade de Medicina da Universidade Federal da Paraíba.

2. Professora-adjunta do Dep. de Medicina da Faculdade de Medicina da Universidade Federal da Bahia.

3. Professor Livre-Docente do Dep. de Medicina da Faculdade de Medicina da UFB.

Fontes financiadoras: CAPES, CNPq, Hospital Universitário Alcides Carneiro - Universidade Federal da Paraíba, Secretaria de Saúde de Campina Grande.

Artigo submetido em 02.08.02, aceito em 05.02.03.

\begin{abstract}
Objective: to assess the relation between children with asthma and ascariasis in the low income neighborhood of Pedregal, in Campina Grande (Paraíba, Brazil).

Material and method: the International Study of Asthma and Allergies in Childhood (ISSAC) standard questionnaire was distributed to 1,095 children but only 742 of them answered it and provided stool samples for parasite examination. The cross-sectional study included those 742 children, whose age ranged from 2 to 10 years. The association between asthma and ascariasis was studied through Kappa rating. Demographic and clinic variants comparing the two groups were analyzed by chi-square test $\left(\chi^{2}\right)$ or $t$ Student test.

Results: among the 742 children analyzed, 51.9\% (385) were males and $48.1 \%$ (357) females. The prevalence of asthma was $59.7 \%(443)$ and that of ascariasis was $56.3 \%$ (418). The frequency of asthma in the ascariasis group $(60.5 \%)$ was similar $\left(\chi^{2}=0.27\right.$, $\mathrm{p}>0.06)$ to the frequency of asthma in the non-infected group $(58.6 \%)$.
\end{abstract}

Conclusion: despite the high prevalence of the diseases studied, there is no apparent relation between asthma and ascariasis in the population studied.

JPediatr (Rio J) 2003;79(3):227-32: asthma, ascariasis, children, prevalence.

\section{Introdução}

A asma é uma doença crônica muito freqüente em crianças, traduzida pelos episódios de sibilância pulmonar, e a sua gravidade vem aumentando em vários países do mundo $^{1}$. Como se sabe, a predisposição genética é o fator de risco mais forte, mas muitos outros participam, como fatores ambientais, hábitos alimentares, tabagismo, industrialização e aumento da poluição do ar nas grandes metró- 
poles $^{2}$. A prevalência de asma no Brasil tem sido estudada ${ }^{3}$, utilizando-se o questionário padronizado do International Study of Asthma and Allergies in Childhood (ISAAC). Em crianças de Recife, Salvador, São Paulo, Curitiba e Porto Alegre, a prevalência de "sibilos alguma vez" variou de $39,0 \%$ a $47,0 \% 4$.

Por outro lado, a ascaridíase é uma das parasitoses mais freqüentes no Brasil, especialmente nas áreas rurais, onde foram observadas prevalências de 74,8\% e de 75,2\%, respectivamente, por Lucena \& Magalhães Neto e Pereira et al., em Pernambuco ${ }^{5}$. Em outra população de Campina Grande, com características sociais semelhantes, Pereira et al. ${ }^{11}$ encontraram prevalência de ascaridíase de 54,2\%. Ainda no Nordeste, nas grandes metrópoles (Recife), com a ocupação urbana caótica das periferias, sem estrutura sanitária básica, acredita-se que ocorra o aumento significativo das enteroparasitoses, com prevalências de $56 \%$ a $76,8 \%{ }^{5}$.

A possível associação entre asma e ascaridíase foi proposta por Lynch et al. (1998), estudando dois grupos de crianças do mesmo nível socioeconômico, e com igual exposição a helmintos, diferenciando-os apenas pela prevalência de asma ${ }^{6}$. Apesar das mesmas condições de vida, a intensidade da infecção parasitária foi maior nas crianças não alérgicas. Em trabalho anterior ${ }^{7}$, foi observado que, em áreas endêmicas de ascaridíase e após tratamento com antihelmínticos, há diminuição dos níveis séricos de IgE total e de IL-4, em contraste com a elevação dos níveis séricos de IgE específica, bem como da reatividade cutânea nas pessoas alérgicas.

Apesar da relevância médico-social da asma e da ascaridíase em população de crianças brasileiras, não foi encontrado nenhum outro estudo mostrando se há ou não associação destes dois agravos à saúde. Em Campina Grande (PB), as elevadas freqüências desses agravos motivaram o estudo da associação entre asma e ascaridíase descrita na literatura ${ }^{6,7}$.

\section{Material e métodos}

A população estudada foi constituída por crianças de dois a 10 anos de idade, residentes no bairro do Pedregal, da cidade de Campina Grande (PB). Conforme dados fornecidos pela Secretaria Municipal de Saúde, existem 2.655 famílias cadastradas pelo Programa de Saúde da Família (PSF), sendo a população estimada de 10.706 habitantes. Deste total, a estimativa é que as crianças na faixa etária de 2 a 10 anos compreendem $14,9 \%$ ( $\cong$ @1.600) dos moradores.

O trabalho foi iniciado em $1^{\circ}$ de julho e concluído em 30 de outubro de 2001. Empregou-se o questionário escrito padrão do ISAAC (módulo asma) para identificar os possíveis asmáticos. Ele foi aplicado aos responsáveis de 1.095 crianças durante visita domiciliar, quando foram entregues os recipientes para a coleta de fezes. Antes do preenchimen- to do questionário padrão ISAAC ${ }^{3}$, o termo de consentimento livre e esclarecido era lido e explicado, até que não houvessem dúvidas por parte do responsável legal. Foram incluídas $742(67,8 \%)$ crianças, que entregaram as amostras fecais para realização do exame parasitológico no laboratório de patologia clínica do Hospital Universitário Alcides Carneiro (HUAC) da Universidade Federal da Paraíba (Campina Grande, PB). Portanto, foram perdidas, ou não participaram pelo fechamento do posto de coleta do bairro, $353(32,2 \%)$ crianças, por não entregarem o espécime fecal para o exame parasitológico. O técnico responsável pelo exame parasitológico desconhecia a qual grupo pertencia a criança (asma ou não-asma).

O questionário padrão do $\mathrm{ISAAC}^{3}$ foi aplicado para avaliar a prevalência da asma e seus sintomas, sendo caracterizadas como portadoras de asma aquelas crianças com a presença de sibilância em algum período de sua vida. $\mathrm{Na}$ pesquisa de ovos do Ascaris lumbricoides nas fezes, foi utilizado o método de sedimentação espontânea de Lutz, Hoffman, Pons e Janner ${ }^{8}$. Todas as crianças com ovos de geo-helmintos foram tratadas com mebendazol (100mg, $2 \mathrm{x}$ por dia, durante três dias), bem como os portadores de outras parasitoses intestinais diagnosticadas.

Nas análises estatísticas das variáveis avaliadas (sexo, idade, renda familiar, história familiar de atopia, diagnóstico de asma e de ascaridíase), foi usado o software SPSS (Statistical Packge for Social Sciences) versão 9.0 for Windows. Na avaliação da concordância (associação) dos diagnósticos de asma e da infecção pelo A. lumbricoides, foi aplicado o índice de Kappa. Na análise das demais variáveis, conforme o tipo das mesmas, foram aplicados o teste do qui-quadrado $\left(\chi^{2}\right)$ ou o teste $t$ de Student, para amostras pareadas. Na interpretação dos testes estatísticos, foi considerado como de significância estatística se a probabilidade (p) de erro tipo I $(\alpha)$ fosse igual ou inferior a $5,0 \%(\mathrm{p} \leq 0,05)$.

\section{Resultados}

Nas 742 crianças, a média de idade foi de 5,6 $\pm 2,7$ anos, sendo 51,9\% (n=385) do sexo masculino e 48,1\% (n=357) do feminino. Como mostra a Tabela 1, a faixa etária predominante $(32,1 \%)$ foi de 8 a 10 anos, e a maioria $(56,5 \%)$ das famílias tinha renda mensal de um saláriomínimo.

A prevalência observada de ascaridíase foi de $56,3 \%$ (418/742), e a da asma foi de 59,7\% (443/742). No conjunto das crianças incluídas inicialmente no estudo $(\mathrm{n}=1.095)$, a freqüência de asma foi de 60,5\% (663/1.095), e entre aquelas posteriormente excluídas, foi de 62,3\% (220/353), mas essas diferenças não foram estatisticamente significantes $\left(\chi^{2}=0,69 ; \mathrm{p}>0,70 ; \mathrm{g} .1=1\right)$.

A Tabela 1 também mostra, dentro de cada grupo, quanto ao resultado do questionário ISSAC (com ou sem asma), que a distribuição da ascaridíase não diferiu ( $p>0,28)$ 
quanto ao sexo das crianças, média de idade, faixa etária ou renda familiar. No entanto, no grupo sem asma, as meninas com ascaridíase $(60,1 \%)$ foram mais freqüentes que os meninos $(49,3 \%)$, mas essa diferença ficou no limite da significância estatística $(\mathrm{p}=0,06)$.

Em relação à asma e seus sintomas, como mostra a Tabela 2, a freqüência de "sibilos alguma vez na vida" foi de 59,7\% (443/742); "sibilos nos últimos 12 meses", de 34,8\% (258/742); "número de crises", 13,7\% (101/742) apresentaram mais de 12 crises no ano; "dificuldade na fala" foi de 31,0\% (229/742); "sibilos após exercício", de $29,4 \%$ (218/742) e "tosse noturna", de 62,7\% (465/742). Esses sintomas não diferiram, estatisticamente $(\mathrm{p}>0,20)$, entre o grupo com ou sem ascaridíase.

Ao comparar a concordância $(52,2 \% ; 387 / 742)$ dos diagnósticos de asma e ascaridíase (em 253 crianças) e a ausência de ambos (em 134 crianças) com a discordância $(47,8 \% ; 355 / 742)$ dos diagnósticos (165 crianças com ascaridíase e sem asma, e 190 com asma e sem ascaridíase), o índice Kappa foi de 0,019 - considerado dentro dos limites de concordância fraca ou muito baixa $(\mathrm{k}$ de $0,0 \mathrm{a}$ $0,20)$.

Avaliando a história de alergia na família (Tabela 3), as freqüências de pais, avós e tios com história semelhante não diferiu ( $p>0,07)$ entre os grupos de crianças com ou sem asma. Porém, as crianças portadoras de asma apresentaram, significativamente $(p<0,0001)$, freqüência maior $(68,2 \%)$ de irmãos com história de alergia, ao ser comparada a frequiência $(31,8 \%)$ naqueles sem a mesma história.

\section{Discussão}

A asma e a ascaridíase são consideradas agravos freqüentes em todo o mundo. No Brasil, onde as condições socioeconômicas interferem diretamente na qualidade de vida das pessoas, expondo-as a todos os tipos de risco e exacerbando, cada vez mais, a pobreza e o dualismo social, a elevada prevalência $(56,3 \%)$ de ascaridíase na comunidade do bairro do Pedregal pode ser outro indicador sobre as precárias condições sociais e de saneamento. Além disso, essa prevalência é superior às encontradas em Curitiba $(42,7 \%)^{9}$ e Salvador $(31,2 \%)^{10}$, e também em estudo anterior, realizado em Campina Grande $(54,2 \%)^{11}$.

Por sua vez, a asma é estudada em todo o mundo, devido não só à sua elevada prevalência, mas também porque é muito freqüente na população infantil de países mais desenvolvidos ${ }^{15}$. No Brasil, a prevalência de asma em crianças de outras cidades brasileiras (Porto Alegre, Curitiba, São Paulo, Uberlândia, Itabira e Recife $)^{16}$ foi inferior a 50\%, sendo a mais elevada em São Paulo, com $49,3 \%{ }^{16}$. Isto, portanto, evidencia que a prevalência de asma observada $(59,7 \%)$ nas crianças do bairro do Pedregal (Campina Grande, Paraíba) além de elevada, acomete população extremamente vulnerável a outros agravos e com dificuldades de acesso aos serviços de saúde.

Tanto a ascaridíase como a asma, nas crianças estudadas, apresentavam distribuição homogênea em relação às variáveis estudadas. Ou seja, não houve diferença significativa nas freqüências dos dois agravos estudados em relação ao sexo, à idade e ao estrato socioeconômico, expresso pela

Tabela 1 - Características demográficas da população infantil do bairro Pedregal, de Campina Grande (PB), distribuída conforme a presença de ascaridíase e de asma

\begin{tabular}{|c|c|c|c|c|c|c|c|c|c|c|c|}
\hline \multirow{3}{*}{\multicolumn{2}{|c|}{$\begin{array}{l}\text { Características } \\
\text { demográficas }\end{array}$}} & \multicolumn{8}{|c|}{ ASMA } & \multirow{3}{*}{ Total } & \multirow{3}{*}{$(\%)$} \\
\hline & & \multicolumn{4}{|c|}{ SIM } & \multicolumn{4}{|c|}{ NÃO } & & \\
\hline & & $\mathbf{n}$ & Áscaris + & $(\%)$ & $\mathbf{p}$ & $\mathbf{n}$ & Áscaris + & $(\%)$ & $\mathbf{p}$ & & \\
\hline Sexo & $\begin{array}{l}\mathrm{n} \\
\text { Masculino } \\
\text { Feminino }\end{array}$ & $\begin{array}{l}443 \\
249 \\
194\end{array}$ & $\begin{array}{l}253 \\
145 \\
108\end{array}$ & $\begin{array}{l}(57,1) \\
(58,2) \\
(55,7)\end{array}$ & $>0,58^{*}$ & $\begin{array}{l}299 \\
136 \\
163\end{array}$ & $\begin{array}{c}165 \\
67 \\
98\end{array}$ & $\begin{array}{l}(55,2) \\
(49,3) \\
(60,1)\end{array}$ & $=0,06$ & $\begin{array}{l}742 \\
385 \\
357\end{array}$ & $\begin{array}{r}(100,0) \\
(51,9) \\
(48,1)\end{array}$ \\
\hline $\begin{array}{l}\text { Idade } \\
\text { (anos) }\end{array}$ & $\begin{array}{l}\text { Média } \\
\mathrm{DP}( \pm)\end{array}$ & $\begin{array}{l}5,58^{\S} \\
2,71^{\S}\end{array}$ & $\begin{array}{l}5,85 \\
2,49\end{array}$ & $\begin{array}{l}- \\
-\end{array}$ & $>0,28^{\dagger}$ & $\begin{array}{l}6,16^{\S} \\
2,53^{\S}\end{array}$ & $\begin{array}{l}6,22 \\
2,44\end{array}$ & $\begin{array}{l}- \\
-\end{array}$ & $>0,83$ & $\begin{array}{l}5,6 \\
2,7\end{array}$ & \\
\hline $\begin{array}{l}\text { Faixa } \\
\text { etária } \\
(\text { anos })\end{array}$ & $\begin{array}{l}2|-| 3 \\
4|-| 5 \\
6|-| 7 \\
8|-| 10\end{array}$ & $\begin{array}{l}114 \\
96 \\
98 \\
135\end{array}$ & $\begin{array}{l}58 \\
54 \\
62 \\
79\end{array}$ & $\begin{array}{l}(50,9) \\
(56,2) \\
(63,2) \\
(58,5)\end{array}$ & $>0,32^{*}$ & $\begin{array}{l}57 \\
58 \\
81 \\
103\end{array}$ & $\begin{array}{l}29 \\
34 \\
46 \\
56\end{array}$ & $\begin{array}{l}(50,9) \\
(58,6) \\
(56,8) \\
(54,4)\end{array}$ & $>0,84$ & $\begin{array}{l}171 \\
154 \\
179 \\
238\end{array}$ & $\begin{array}{l}(23,0) \\
(20,8) \\
(24,1) \\
(32,1)\end{array}$ \\
\hline $\begin{array}{l}\text { Renda } \\
\text { familiar } \\
(\ddagger)\end{array}$ & $\begin{array}{l}\text { sem renda } \\
<1 \\
=1 \\
>1\end{array}$ & $\begin{array}{l}37 \\
84 \\
240 \\
82\end{array}$ & $\begin{array}{c}23 \\
53 \\
129 \\
48\end{array}$ & $\begin{array}{l}(62,2) \\
(63,1) \\
(53,8) \\
(58,5)\end{array}$ & $>0,42^{*}$ & $\begin{array}{l}30 \\
32 \\
179 \\
58\end{array}$ & $\begin{array}{l}19 \\
20 \\
99 \\
27\end{array}$ & $\begin{array}{l}(63,3) \\
(62,5) \\
(55,3) \\
(46,6)\end{array}$ & $>0,35^{*}$ & $\begin{array}{l}67 \\
116 \\
419 \\
140\end{array}$ & $\begin{array}{r}(9,0) \\
(15,6) \\
(56,5) \\
(18,9\end{array}$ \\
\hline
\end{tabular}

* Qui-quadrado; † teste $t$ de Student; ‡ em salários-mínimos; § grupo áscaris-negativo. 
Tabela 2 - Sintomas associados à asma em crianças do bairro do Pedregal, de Campina Grande (PB), distribuídos conforme a presença de ascaridíase

\begin{tabular}{|c|c|c|c|c|c|c|c|c|}
\hline \multirow{3}{*}{ Questionário ISSAC } & & \multicolumn{6}{|c|}{ Ascaridíase } & \multirow{3}{*}{$\mathbf{p}$} \\
\hline & & \multicolumn{2}{|c|}{ Sim } & \multicolumn{2}{|c|}{ Não } & \multicolumn{2}{|c|}{ Total } & \\
\hline & & $\mathbf{n}$ & $(\%)$ & $\mathbf{n}$ & $(\%)$ & $\mathbf{n}$ & $(\%)$ & \\
\hline \multirow[t]{2}{*}{ Sibilos 1 vez } & Sim & 253 & $(57,1)$ & 190 & $(42,9)$ & 443 & $(59,7)$ & \multirow[t]{2}{*}{$>0,60^{*}$} \\
\hline & Não & 165 & $(55,2)$ & 134 & $(44,8)$ & 299 & $(40,3)$ & \\
\hline \multirow[t]{2}{*}{ Sibilos no último ano } & Sim & 143 & $(55,4)$ & 115 & $(44,6)$ & 258 & $(34,8)$ & \multirow[t]{2}{*}{$>0,71^{*}$} \\
\hline & Não & 275 & $(56,8)$ & 209 & $(43,2)$ & 484 & $(65,2)$ & \\
\hline \multirow[t]{2}{*}{12 crises no ano } & Sim & 51 & $(50,4)$ & 50 & $(49,6)$ & 101 & $(13,6)$ & \multirow[t]{2}{*}{$>0,20^{*}$} \\
\hline & Não & 367 & $(57,3)$ & 274 & $(42,7)$ & 641 & $(86,4)$ & \\
\hline \multirow[t]{2}{*}{ Dificuldade na fala } & Sim & 125 & $(54,6)$ & 104 & $(45,4)$ & 229 & $(31,0)$ & \multirow[t]{2}{*}{$>0,52^{*}$} \\
\hline & Não & 293 & $(57,1)$ & 220 & $(42,9)$ & 513 & $(69,0)$ & \\
\hline \multirow[t]{2}{*}{ Sibilos após exercício } & Sim & 117 & $(53,7)$ & 101 & $(46,3)$ & 218 & $(29,4)$ & \multirow[t]{2}{*}{$>0,34^{*}$} \\
\hline & Não & 301 & $(57,5)$ & 223 & $(42,5)$ & 524 & $(70,6)$ & \\
\hline \multirow[t]{2}{*}{ Tosse seca noturna } & Sim & 154 & $(33,1)$ & 123 & $(26,5)$ & 465 & $(62,7)$ & \multirow[t]{2}{*}{$>0,75^{*}$} \\
\hline & Não & 264 & $(95,3)$ & 201 & $(72,6)$ & 277 & $(37,3)$ & \\
\hline
\end{tabular}

*Qui-quadrado.

renda familiar. Não obstante, há relatos na literatura descrevendo a asma como mais freqüente em meninos ${ }^{12}$, de predominância entre seis a sete anos de idade ${ }^{13}$, e entre aquelas famílias mais carentes ou de menor renda famili$\operatorname{ar}^{14-15}$.

Desse modo, a não observação da maior freqüência de ascaridíase entre as crianças do sexo masculino, de maior idade, e de famílias de menor renda induz à especulação de que os riscos de infecção pelo A. lumbricoides são comuns nos ambientes intra e extradomiciliar do bairro do Pedregal, onde as condições sanitárias são próprias dos locais com baixos indicadores de desenvolvimento humano. Isto porque, caso a ascaridíase predominasse entre meninos, de maior idade e de famílias mais carentes, poder-se-ia explicar por serem essas as características facilitadoras à maior exposição dos meninos aos fatores ambientais, extradomiciliares, nos quais a poluição ambiental é habitual, e, conseqüentemente, a contaminação de larvas do A. lumbri-

Tabela 3 - Distribuição da história familiar de alergia em parentes consangüíneos de crianças do bairro Pedregal de Campina Grande (PB)

\begin{tabular}{|c|c|c|c|c|c|c|c|c|}
\hline \multirow{3}{*}{ História da alergia } & & \multicolumn{6}{|c|}{ Asma na criança } & \multirow{3}{*}{$\mathbf{p}$} \\
\hline & & \multicolumn{2}{|c|}{ Sim } & \multicolumn{2}{|c|}{ Não } & \multicolumn{2}{|c|}{ Total } & \\
\hline & & $\mathbf{n}$ & $(\%)$ & $\mathbf{n}$ & $(\%)$ & $\mathbf{n}$ & $(\%)$ & \\
\hline Pai & $\begin{array}{l}\text { Sim } \\
\text { Não }\end{array}$ & $\begin{array}{l}28 \\
415\end{array}$ & $\begin{array}{l}(66,7) \\
(59,3)\end{array}$ & $\begin{array}{l}14 \\
285\end{array}$ & $\begin{array}{l}(33,4) \\
(40,7)\end{array}$ & $\begin{array}{l}42 \\
700\end{array}$ & $\begin{array}{l}(5,7) \\
(94,3)\end{array}$ & $>0,34^{*}$ \\
\hline Mãe & $\begin{array}{l}\text { Sim } \\
\text { Não }\end{array}$ & $\begin{array}{l}61 \\
382\end{array}$ & $\begin{array}{l}(64,9) \\
(59,0)\end{array}$ & $\begin{array}{l}33 \\
266\end{array}$ & $\begin{array}{l}(35,1) \\
(41,0)\end{array}$ & $\begin{array}{l}94 \\
648\end{array}$ & $\begin{array}{l}(12,7) \\
(87,3)\end{array}$ & $>0,27^{*}$ \\
\hline Irmandade & $\begin{array}{l}\text { Sim } \\
\text { Não }\end{array}$ & $\begin{array}{l}219 \\
224\end{array}$ & $\begin{array}{l}(68,2) \\
(53,2)\end{array}$ & $\begin{array}{l}102 \\
197\end{array}$ & $\begin{array}{l}(31,8) \\
(46,8)\end{array}$ & $\begin{array}{l}321 \\
421\end{array}$ & $\begin{array}{l}(43,3) \\
(56,7)\end{array}$ & $<0,0001^{*}$ \\
\hline Avós & $\begin{array}{l}\text { Sim } \\
\text { Não }\end{array}$ & $\begin{array}{l}76 \\
367\end{array}$ & $\begin{array}{l}(67,3) \\
(58,3)\end{array}$ & $\begin{array}{l}37 \\
262\end{array}$ & $\begin{array}{l}(32,7) \\
(41,7)\end{array}$ & $\begin{array}{l}113 \\
629\end{array}$ & $\begin{array}{l}(15,2) \\
(84,8)\end{array}$ & $>0,07^{*}$ \\
\hline Tios & $\begin{array}{l}\text { Sim } \\
\text { Não }\end{array}$ & $\begin{array}{l}108 \\
335\end{array}$ & $\begin{array}{l}(57,4) \\
(60,5)\end{array}$ & $\begin{array}{l}80 \\
219\end{array}$ & $\begin{array}{l}(42,6) \\
(39,5)\end{array}$ & $\begin{array}{l}188 \\
554\end{array}$ & $\begin{array}{l}(25,3) \\
(74,7)\end{array}$ & $>0,46^{*}$ \\
\hline
\end{tabular}

* Qui-quadrado. 
coides. Portanto, aquela homogeneidade da população estudada também denuncia as iguais chances de infecção pelo A. lumbricoides e até a uniformidade da falta de acesso aos serviços públicos e de outros bens ou serviços.

Coincidentemente, as elevadas freqüências de sintomas associados à asma, observados nas crianças do bairro do Pedregal, também são comuns em locais de clima quente, úmido e com baixos indicadores socioeconômicos, onde estão presentes grande número de fatores ambientais de risco ${ }^{4}$. Nestas áreas, a forma desordenada como vem se dando a urbanização faz com que aglomerados urbanos cresçam de modo caótico, com infra-estrutura física, habitação e serviços altamente vulneráveis ${ }^{19}$. Nesses ambientes, as infecções gastrintestinais (parasitárias, bacterianas e virais) são consideradas, por alguns autores ${ }^{17}$, como fatores protetores à resposta imune Th1 e, conseqüentemente, funcionando como mecanismos reguladores para a menor ocorrência de asma na população mais exposta àquelas infecções.

No entanto, isto aparentemente não ocorre entre as crianças estudadas, porque, além do elevado percentual de casos de asma, as doenças infectoparasitárias são comuns e, mais ainda, as condições associadas à miséria humana são os maiores fatores predisponentes de agravos à saúde entre aquelas crianças, muito provavelmente, desde o período pré-natal.

Em acordo à suposição de fatores predisponentes à asma da criança, igualmente distribuídos na comunidade estudada, houve significativa associação de casos entre aqueles com irmãos também acometidos de atopias, entre as quais a asma, ao contrário do descrito por Moraes et al. ${ }^{18}$. De outro modo, essa mesma associação não foi observada com os outros membros parentais das crianças, adultos (pais, avós e tios), o que, independente dos fatores genéticos e familiais, até certo modo pode ser explicado pelas diferenças no tipo e na intensidade da exposição ambiental, entre outros fatores, próprios de cada faixa etária ${ }^{14,17,18}$.

Nestas condições, seria até surpreendente a ocorrência maior de casos de asma entre aqueles portadores também de ascaridíase, como descrito por outros autores ${ }^{6,7}$. Como doença multifatorial, a asma é desencadeada ou predisposta por diferentes aeroalérgenos, infecções bacterianas e virais, entre outros fatores ambientais e genéticos ${ }^{2}$. Dessa forma, estimar quais os fatores ambientais estão mais associados à asma iria requer um bem-planejado estudo de coorte, formado por diferentes grupos quanto ao tipo de exposição, na tentativa de explicar qual o melhor modelo para a doença. Mesmo assim, as peculiaridades e multiplicidade de fatores ambientais, inclusive aqueles locais ou regionais, bem como a diversidade da constituição gênica das populações humanas, tornam este tipo de estudo praticamente inexequíivel, mesmo que os recursos disponíveis fossem ilimitados. Assim sendo, e também considerando ser a infecção pelo A. lumbricoides de ocorrência contínua e de intensidade variável ao longo do tempo, havendo condições ambientais favoráveis, outros modelos de estu- do, exceto os transversais, sofrem sérias restrições quanto à exeqüibilidade, especialmente se também considerado o tamanho amostral, a necessária aderência ao estudo e o número de exames parasitológicos para o aumento da sensibilidade do diagnóstico de ascaridíase. Nesse estudo, por exemplo, mesmo que baseado em somente uma amostra fecal e na participação ativa das equipes das unidades de saúde da família do bairro do Pedregal, aproximadamente um terço $(32,2 \%)$ das crianças não foram incluídas no estudo, posto que ou não entregaram a amostra fecal, ou o fizeram imediatamente antes do fechamento do local de coleta no bairro do Pedregal. Apesar disso, segundo os dados populacionais obtidos, mais de $50 \%$ da população alvo foi avaliada.

Em conclusão, apesar da falta de evidências de associação entre a asma e a ascaridíase nas crianças estudadas, as elevadas prevalências observadas desses agravos indicam também a necessidade da adoção de medidas sanitárias urgentes na área de estudo, bem como programas de promoção de saúde e de prevenção de doenças, os quais não só cabem aos três níveis de governo como devem ser necessários a várias outras populações semelhantes do Brasil, especialmente das regiões Norte e Nordeste.

\section{Agradecimentos}

Ao Professor Dirceu Solé, Titular e Livre-Docente em Alergia, Imunologia Clínica e Reumatologia da Escola Paulista de Medicina da Universidade Federal de São Paulo, pelo inestimável apoio para a realização desse estudo.

\section{Referências bibliográficas}

1. Camelo-Nunes IC, Solé D, Naspitz CK. Fatores de risco e evolução clínica da asma em crianças. J Pediatr (Rio J) 1997; 73:151-60.

2. Galvão CES, Kalil J, Castro FM. Sensibilização a aeroalérgenos em dois grupos de escolares na zona rural e urbana de São Paulo, Brasil. Revista Brasileira de Alergia Imunopatologia 2002;25:2-9.

3. Solé D, Vanna AT, Yamada E, Rizzo MC, Naspitz CK. International Study of Asthma and Allergies in Childhood (ISSAC) written questionnaire: validation of the asthma component among Brazilian children. J Investig Allergol Clin Immunol 1998;8:376-82.

4. Mallol J, Solé D, Asher I, Clayton T, Stein R, Soto-Quiroz M. Prevalence of asthma symptoms in Latin America: The International Study of Asthma and Allergies in Childhood (ISSAC). Pediatr Pulmonol 2000;30:439-44.

5. Huggins D, Medeiros LB, Souza UBM, Agnia TCT. Parasitoses intestinais no período infantil. Pediatria Moderna 2000;36: 641-72. 
6. Lynch NR, Hagel I, Palenque ME, Di Prisco MC, Escudero JE, Corao LA, et al. Relation between helmintic infection and IgE response in atopic and nonatopic children in a tropical environment. J Allergy Clin Immunol 1998;101:217-21.

7. Lynch NR, Hagel I, Perez M, Di Prisco MC, Lopez R, Alvarez $\mathrm{N}$. Effect of anthelmintic treatment on the allergic reactivity of children in a tropical slum. J Allergy Clin Immunol 1993;92: 404-11.

8. Pessoa SB, Martins AV. Parasitologia Médica. $11^{\mathrm{a}}$ ed. Rio de Janeiro: Guanabara Koogan; 1982.p.872.

9. Rosário Filho, NA. Níveis séricos da Imunoglobulina E em enteroparasitoses. Efeito do tratamento específico, correlação com contagens de eosinófilos e testes cutâneos para alergia [dissertação]. Curitiba: Universidade Federal do Paraná; 1980.

10. Prado MS, Barreto M, Strina A, Faria JAS, Nobre AA, Jesus SR. Prevalência e intensidade da infecção por parasitas intestinais em crianças na idade escolar na cidade de Salvador (Bahia, Brasil). Revista Brasileira de Medicina Tropical 2000;57:226-36.

11. Pereira G, Corrêa FJV, Porto SMB, Ribeiro FRF, Ferreira MA. A incidência de enteroparasitoses numa das favelas da periferia de Campina Grande-PB. Anais da $47^{\mathrm{a}}$ Reunião Anual da SBPC; 1995 09-14 de julho; São Luís, Brasil. São Luís: SBPC; 1995.

12. Trippia SMG, Rosário NA, Ferreri FP. Aspectos clínicos da asma na criança: análise de 1009 pacientes em um ambulatório especializado. Revista Brasileira de Alergia Imunopatologia 1998;21:75-82.

13. Solé D, Naspitz CK. Epidemiologia da asma: Estudo ISSAC (International Study of Asthma and Allergies in Childhood). Revista Brasileira de Alergia Imunopatologia 1998;21:38-45.
14. Weitzman M, Gortmaker S, Sobol A. Racial, social and environmental risk foe childhood asthma. Am J Dis Child 1990; 144:1189-94.

15. Persky VW, Slezak J, Contreras A, Becker L, Hernandez E, Ramakrishnan V, et al. Relationship of race and socioeconomic status with prevalence, severity and symptoms of asthma in Chicago school children. Ann Allergy Asthma Immunol 1998; 81:266-71.

16. Solé D, Yamada E, Vana AT, Werneck G, Solano de Freitas L, Sologuren MJ, et al. International Study of Asthma and Allergies in Childhood (ISSAC): Prevalence of asthma and asthma-related symptoms among Brazilian schoolchildren. J Investig Allergol Clin Immunol 2001;11:123-8.

17. Correia JMM, Zuliani A. Imunidade relacionada à resposta a doença alérgica. J Pediatr (Rio J) 2001;77:441-6.

18. Moraes LSL, Barros MD, Takano AO, Assami NMC. Fatores de risco, aspectos clínicos e laboratoriais da asma em crianças. J Pediatr (Rio J) 2001;77:447-54.

19. Ramalho DS. Degradação ambiental urbana e pobreza: a percepção dos riscos. Raízes 1999;19:16-30.

Endereço para correspondência:

Dra. Maria Teresa Nascimento Silva

Rua Antonio Joaquim Pequeno, 602 - ap. 203 - Bodocongó CEP 58109-085 - Campina Grande, PB

Tels.: (83) 3331976 / 3223020

E-mail:teresans@terra.com.br 